\title{
Sophocarpine attenuates septic liver injury through suppression of the NLRP3 inflammasome via autophagy-mediated degradation
}

\author{
NIANGUO HOU ${ }^{1}$, XIAOFENG DAI $^{2}$, WENQING LU ${ }^{3}$, HONGGUANG YANG $^{1}$, \\ HAIDA YU ${ }^{1}$, JUNCHAO LIU ${ }^{1}$, HUI LI $^{1}$, XUNJUN SHUAI ${ }^{1}$ and DENGBIN AI ${ }^{1}$ \\ ${ }^{1}$ Department of Anesthesiology, Qingdao Municipal Hospital, Qingdao, Shandong 266011; \\ ${ }^{2}$ Department of Anesthesiology, Qingdao Central Hospital, Qingdao, Shandong 266000; \\ ${ }^{3}$ Department of Anesthesiology, Weifang Medical University, Weifang, Shandong 261053, P.R. China
}

Received November 21, 2019; Accepted April 29, 2020

DOI: 10.3892/etm.2020.9379

\begin{abstract}
Septic liver injury remains a challenge in sepsis treatment. Nucleotide-binding oligomerization domain, leucine rich repeat and pyrin domain containing 3 (NLRP3) inflammasome activation has been suggested to be a major cause of hepatocyte cell death in liver diseases. However, insufficient research has been performed to explore the underlying mechanisms associated with this. In the present study, sophocarpine, a pharmaceutical monomer originally isolated from Sophora flavescens, was suggested to attenuate septic liver injury in a mouse cecal ligation and puncture (CLP) model. By utilizing western blotting, ELISA, H\&E staining and immunohistochemistry, the results demonstrated that sophocarpine treatment reversed CLP-induced elevations in serum aspartate transaminase, alanine transaminase, interleukin (IL)- 6 and IL- $1 \beta$ levels. Additionally, sophocarpine appeared to have suppressed the activation of the NLRP3 inflammasome, as indicated by observed reductions in liver IL-1 $\beta$, NLRP3, caspase 1-p20 and gasdermin D-p30 protein levels. Further investigation suggested that sophocarpine-induced autophagy was essential for this suppression of NLRP3 inflammasome activation, the inhibition of which reversed the protective effects of sophocarpine on CLP-induced liver injury. Collectively, results from the present study suggested a protective role for sophocarpine against septic liver injury, where sophocarpine may suppress NLRP3 inflammasome activation by autophagy-mediated degradation.
\end{abstract}

Correspondence to: Dr Dengbin Ai, Department of Anesthesiology, Qingdao Municipal Hospital, 1 Jiaozhou Road, Qingdao, Shandong 266011, P.R. China

E-mail: aidengbin@yeah.net

Key words: sophocarpine, nucleotide-binding oligomerization domain, leucine rich repeat and pyrin domain containing 3, inflammasome, sepsis, liver injury

\section{Introduction}

Sepsis is one of the leading causes of mortality in the clinic worldwide (1). The global incidence of sepsis is $>18$ million cases per year, with the mortality rate reaching as high as $28-40 \%$ (1). Sepsis is defined as a life-threatening organ dysfunction caused by uncontrolled inflammatory responses, where the resulting septic liver injury and failure are proposed to be among the major causes of mortality (1-3). Septic liver injury has been identified as an independent risk factor for the prediction of mortality in patients in critical care, where previous studies have reported that alleviation of liver injury improved the prognosis and reduced mortality in patients with sepsis (2-4).

During sepsis, inflammatory cytokines released from immune cells bind to receptors on hepatocytes and induce the activation of downstream signaling, resulting in the apoptosis or necrosis of hepatocytes (4-6). Previous studies identified inflammasome activation in hepatocytes as a major cause of hepatocyte cell death and of septic liver injury and failure (7-10). Following activation by pro-inflammatory cytokines, including interleukin (IL)-1 $\beta$ and IL-6, MAPK and NF- $\mathrm{BB}$ signaling are activated in hepatocytes, which promotes the expression of pro-IL-1 $\beta$ and pro-caspase 1 (6). Pro-IL-1 $\beta$ and pro-caspase are then activated by danger signals, including extracellular ATP or reactive oxygen species, leading to the assembly of the nucleotide-binding oligomerization domain, leucine rich repeat and pyrin domain containing 3 (NLRP3) inflammasome and IL-1 $\beta$ production $(9,11)$. IL-1 $\beta$ in turn activates inflammatory signaling in surrounding hepatocytes, further exacerbating injury and cell death (12). Although it has been speculated that inflammasome activation suppression may have protective effects on liver during sepsis, research remains insufficient (11).

Sophocarpine is a pharmaceutical monomer that was originally derived from herbs of the Sophora species, including Sophora flavescens and Sophora alopecuroides, which have been applied in traditional Chinese medicine (13). Similar extracts, known as Kudouzi, are typically used in Chinese medicine for the treatment of fever, inflammation and edema (13). Recent studies suggested that sophocarpine may alleviate lipopolysaccharide (LPS)-induced liver injury 
in mice through suppression of oxidative stress, inflammation and apoptosis (13-15). These protective effects have been proposed to be due to inhibition of the MAPK and NF- $\kappa \mathrm{B}$ pathways and reductions in the levels of inducible nitric oxide synthase and cyclooxgenase-2 (13). However, to the best of our knowledge, the effects of sophocarpine on sepsis-induced liver injury and inflammasome activation in hepatocytes during sepsis remains poorly understood.

In the present study, the potential effects of sophocarpine on septic liver injury were investigated in a cecal-ligation and puncture (CLP) model, a classical model of murine sepsis (4). In addition, the possible effects of sophocarpine on inflammasome activation in the liver and associated regulatory mechanisms were assessed.

\section{Materials and methods}

Animal model and experiments. A total of 86 male C57BL/6J mice (age, 8-10 weeks; weight, 20-25 g) were purchased from the Qingdao Laboratory Animal Center. The mice were raised in a specific pathogen-free room at a temperature of $18-22^{\circ} \mathrm{C}$, humidity of $50-60 \%$ and on a 12 -h light/dark cycle. The mice received water and food ad libitum. Animal experiments in the present study were approved by the Ethics Committee of Qingdao Municipal Hospital (Qingdao, China). The authors confirm that the animals received humane care and that all animal experiments were performed in accordance with the relevant guidelines and regulations (4). The CLP mouse model was established as previously reported (16). Mice were administered with sophocarpine through oral gavage for 3 days. On the day of CLP procedure, mice were firstly treated with Bafilomycin A or MG132 $2 \mathrm{~h}$ before the CLP procedure. Then mice were subjected to the CLP procedure. Mice were anesthetized using 4\% sevoflurane (Abbott Pharmaceutical Co., Ltd.) and positioned on a sanitized surgical plate. The mouse cecum was isolated and a $0.5 \mathrm{~cm}$ section of cecum was ligated and punctured using a $22 \mathrm{G}$ sterile syringe. NC represents the normal control group. After the CLP procedure, mice were treated with sophocarpine for 3 more days and then sacrificed for tissues collection. Sophocarpine treatment (30 or $60 \mathrm{mg} / \mathrm{kg}$ ) was adapted from a previous study on oral administration (13). To evaluate the effects of sophocarpine on septic liver injury, CLP model mice were treated with sophocarpine at 30 or $60 \mathrm{mg} / \mathrm{kg}$. Mice that underwent the CLP procedure were administered sophocarpine orally (oral gavage) for 2 days pre-operatively and then for 3 days following the CLP-procedure. The mice were sacrificed on day 6 and blood and liver samples were obtained. Bafilomycin A $(10 \mathrm{mg} / \mathrm{kg})$ (16) or MG-132 (10 $\mu \mathrm{g} / \mathrm{kg}$; MedChemExpress), according to previously proposed dosages (17), were injected intraperitoneally $2 \mathrm{~h}$ before the CLP procedure (these inhibitor is administered at the operation day). After the CLP procedure, mice were administered sophocarpine for the following 3 days. The liver was harvested on the fourth day after the CLP procedure. For sample collection mice were first anesthetized using $4 \%$ sevoflurane and then sacrificed by cervical dislocation, according to a previously reported sacrifice method (18).

Analysis of liver function. Serum levels of alanine transaminase (ALT) and aspartate transaminase (AST) were measured to indicate liver function using biochemical kits (AST kit, cat. no. C010-2-1; ALT kit, cat. no. C009-2-1) supplied by Nanjing Jiancheng Bioengineering Institute.

Western blot analysis. Liver tissues or cells were lysed in immunoprecipitation assay buffer (1 mM EDTA, pH 8.0; $50 \mathrm{mM}$ Tris-HCl, $\mathrm{pH} 8.0 ; 2 \%$ sodium dodecyl sulfate; $5 \mathrm{mM}$ dithiothreitol). The protein concentration was then determined using a bicinchoninic acid protein assay kit (Thermo Fisher Scientific, Inc.). Protein samples $(30 \mu \mathrm{g})$ were then subjected to $10 \%$ SDS-PAGE, transferred to PVDF membranes (Invitrogen; Thermo Fisher Scientific, Inc.) and blocked with $5 \%$ non-fat dry milk in phosphate-buffered saline with Tween-20, pH 7.5 at room temperature. The membranes were then incubated with the primary antibodies (dilution 1:2,000) for $4 \mathrm{~h}$ at room temperature followed by incubation with horseradish peroxidase-conjugated secondary antibodies (dilution 1:5,000; cat. nos. 7074 and 7076; Cell Signaling Technology, Inc.) for $3 \mathrm{~h}$ at room temperature. The primary antibodies used in the present study were as follows: Anti-mouse NLRP3 (cat. no. ab214185; rabbit; Abcam), pro-IL-1 $\beta$ (cat. no. 12426; rabbit; Cell Signaling Technology, Inc.), caspase-1 (cat. no. AG-20B-0042-C100; mouse; Adipogen Life Sciences, Inc.), gasdermin D (GSDMD; cat. no. sc393656; mouse; Santa Cruz Biotechnology, Inc.), $\beta$-actin (cat. no. ab8227; rabbit; Abcam), microtubule associated protein 1 light chain 3 (LC3; cat. no. 2775; rabbit; Cell Signaling Technology, Inc.) and p62 (cat. no. 5114; rabbit; Cell Signaling Technology, Inc.). Signal intensity was determined using a Chemiluminescent Imaging System 5200S (Tanon Science and Technology Co., Ltd.).

Hematoxylin and eosin, immunohistochemical and immunofluorescence (IF) histochemistry (IFHC). Mouse liver tissues were fixed at room temperature for $24 \mathrm{~h}$ with $10 \%$ formalin and processed into paraffin blocks and sectioned into $5-\mu \mathrm{m}$ slices. Tissues were stained with H\&E for histological analysis according to the standard protocol by previous proposed method (8) and subjected to microscopy by Nikon N1 (Japan). All tissues were examined by three images and subjected to analysis.

For TUNEL staining, section slides were washed with PBS and then subjected to dewaxing in a descending graded ethanol series. TUNEL staining of liver sections was conducted using an In Situ Cell Death Detection kit, according to the manufacturer's protocol (Roche Diagnostics). The slides were then subjected to microscopy (Nikon Corporation) for image capture.

For IFHC, the sections were deparaffinized and hydrated and underwent antigen retrieval using a retrieval solution (EDTA Antigen Retrieval Solution; Beijing Solarbio Science \& Technology Co., Ltd), according to the manufacturer's protocol (Beyotime Institute of Biotechnology). Following blocking with normal goat serum for $1 \mathrm{~h}$ at room temperature, the sections were stained for NLRP3 (dilution 1:500) at room temperature for $3 \mathrm{~h}$ (cat. no. ab214185; Abcam). In total, $\leq 3$ hepatic lobules in three sections per liver tissue from three mice of each group were observed and evaluated by IF staining. The fluorescent secondary antibody used was Alexa ${ }^{\circledR}$ 488-conjugated donkey anti-rabbit at room temperature for $3 \mathrm{~h}$ along with a mounting medium (NS2832, Molecular Probes; 

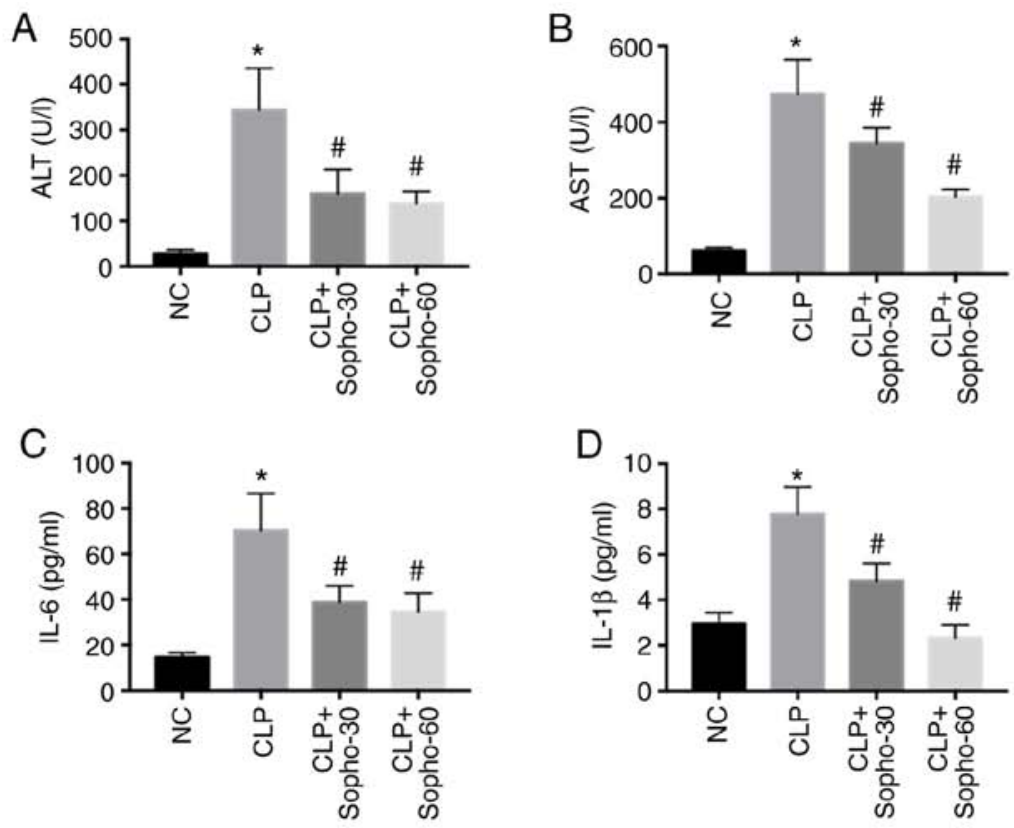

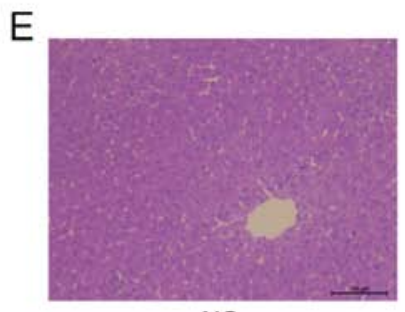

NC

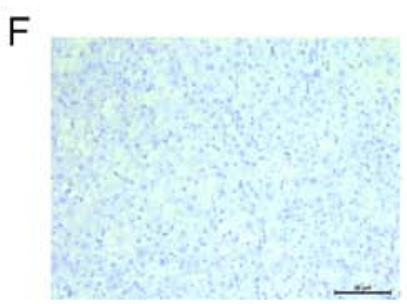

NC

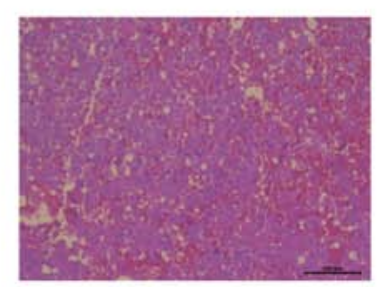

CLP

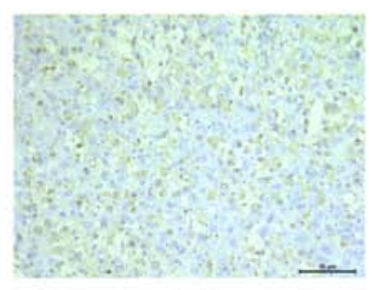

CLP

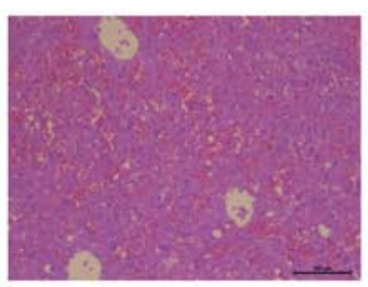

CLP+Sopho-30

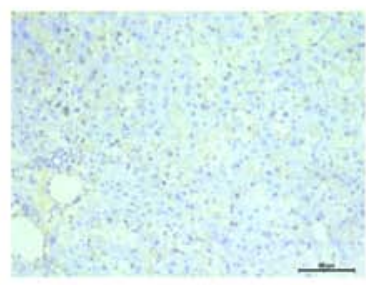

CLP+Sopho-30

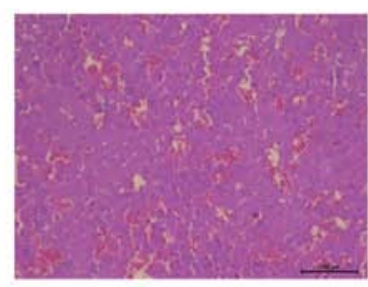

CLP+Sopho-60

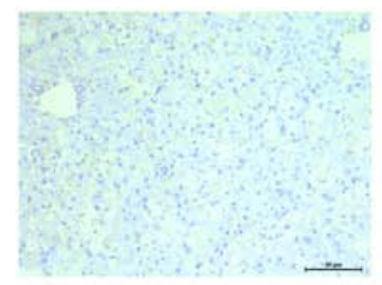

CLP+Sopho-60

Figure 1. Sophocarpine attenuates septic liver injury in a dose-dependent manner. Serum levels of (A) ALT, (B) AST, (C) IL-6 and (D) IL-1 $\beta$ were measured in $\mathrm{NC}, \mathrm{CLP}$ or mice that underwent CLP followed by treatment with 30 or $60 \mathrm{mg} / \mathrm{kg}$ sophocarpine. $\mathrm{n}=5 \mathrm{mice}$ per group. Representative images of (E) hematoxylin and eosin and (F) TUNEL staining of liver sections. Scale bar, $50 \mu \mathrm{m}$. ${ }^{*} \mathrm{P}<0.05$ vs. NC and ${ }^{\#} \mathrm{P}<0.05$ vs. CLP. ALT; alanine transaminase; AST, aspartate transaminase; CLP, cecal ligation and puncture; IL, interleukin; NC, normal control; Sopho-30, sophocarpine treatment 30 mg/kg; Sopho-60, sophocarpine treatment $60 \mathrm{mg} / \mathrm{kg}$.

Invitrogen; Thermo Fisher Scientific, Inc.). Nuclei were counterstained at room temperature using DAPI for 2 min followed by washing with PBS (Sigma-Aldrich; Merck KGaA). The slides were then subjected to fluorescence microscopy using a Nikon N1 microscope (Nikon Corporation) at a magnification of x200 and processed with a cooled camera and NIS Viewer software (version 1.2; Nikon Corporation).

IL-1 $\beta$ and IL-6 enzyme-linked immunosorbent assay (ELISA). The levels of IL-1 $\beta$ of in liver homogenates and the levels of IL- 6 and IL-1 $\beta$ in serum were analyzed. The liver tissues were first weighed and incubated in cold PBS containing $2 \mathrm{mM}$ phenylmethylsulfonyl fluoride, $0.1 \mathrm{mg} / \mathrm{ml}$ trypsin inhibitor, $10 \mathrm{nM}$ EDTA, $0.002 \%$ sodium azide and $1.0 \mathrm{mg} /$ ml BSA (Beyotime Institute of Biotechnology). The tissues were then homogenized and incubated for $2 \mathrm{~h}$ at $4^{\circ} \mathrm{C}$. Samples were then centrifuged at $12,000 \mathrm{xg}$ for $10 \mathrm{~min}$ at $4^{\circ} \mathrm{C}$, where the supernatant was subjected to the IL-1 $\beta$ ELISA (mouse IL-1 $\beta /$ IL-1F2 Quantikine ELISA kit; cat. no. MLB00C; R\&D Systems, Inc.). Serum samples were prepared by centrifuging the blood samples at $4^{\circ} \mathrm{C}$ with $300 \mathrm{x}$ g for $15 \mathrm{~min}$ and collected as previous described (7). The analysis of the IL-6 (mouse IL-6 Quantikine ELISA kit; cat. no. M6000B) and IL-1 $\beta$ levels (mouse IL-1 $\beta /$ IL-1F2 Quantikine ELISA kit; cat. no. MLB00C) was also performed using ELISA, according to manufacturer's protocols (R\&D Systems, Inc.).

Statistical analysis. Data in the present study are presented as the mean \pm standard deviation. Student's t-tests were used where a comparison was made between two groups. One-way ANOVA was used where a comparison was made between multiple groups. Dunnett's post hoc test was used to compare 

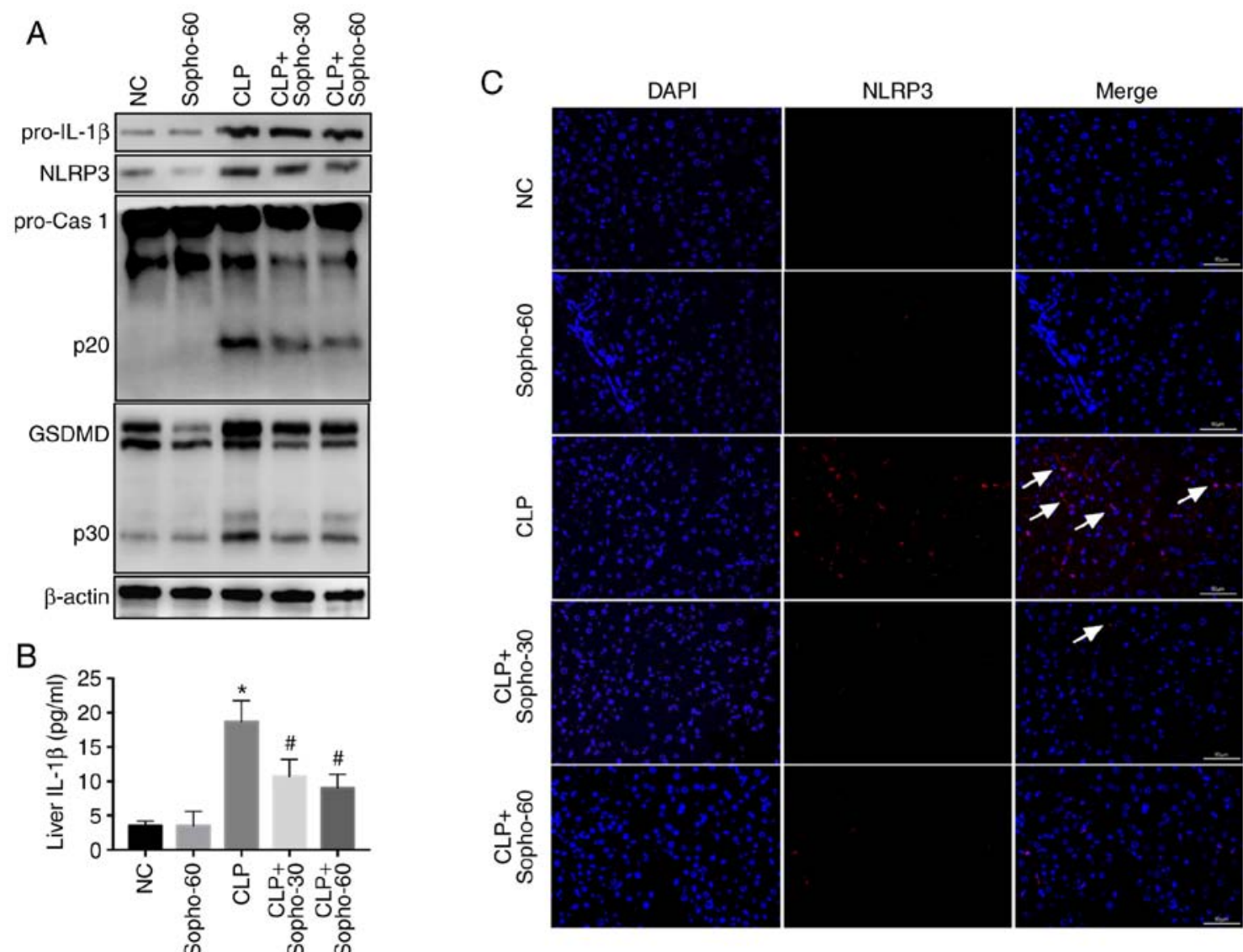

Figure 2. Sophocarpine attenuates NLRP3 inflammasome activation following CLP-induced liver injury. (A) Representative western blotting images of pro-IL-1 $\beta$, NLRP3, pro-caspase 1 and p20, GSDMD and p30 and $\beta$-actin. (B) IL-1 $\beta$ quantification in liver tissues isolated from NC, CLP model and sophocarpine-treated mice. $n=5$ mice per group. (C) Immunofluorescence of NLRP3 staining in liver tissues isolated from mice in the respective treatment groups. Scale bar, $50 \mu \mathrm{m}$. "P $<0.05$ vs. NC and ${ }^{\#} \mathrm{P}<0.05$ vs. CLP. CLP, cecal ligation and puncture; cas, caspase; GSDMD, gasdermin D; IL, interleukin; NC, normal control; Sopho-30, sophocarpine treatment $30 \mathrm{mg} / \mathrm{kg}$; Sopho- 60 , sophocarpine treatment $60 \mathrm{mg} / \mathrm{kg}$; NLRP3, nucleotide-binding oligomerization domain, leucine rich repeat and pyrin domain containing 3 . White arrows indicate inflammasome activation induced by NLRP3 staining.

all groups to the control and Šidák's multiple comparisons test was used for comparison between groups. Statistical analysis was performed using Prism software (version 7; GraphPad Software, Inc.). $\mathrm{P}<0.05$ was considered to indicate a statistically significant difference.

\section{Results}

Sophocarpine attenuates CLP-induced liver injury in a dose-dependent manner. Mice that underwent CLP exhibited significantly elevated serum AST, ALT, IL-6 and IL-1 $\beta$ levels compared with those in the NC group (Fig. 1A-D). However, in the groups treated with sophocarpine at both doses tested (30 and $60 \mathrm{mg} / \mathrm{kg}$ ), AST, ALT, IL-6 and IL-1 $\beta$ levels were all found to be significantly reduced compared with those in the CLP group, with a greater reduction observed when the higher sophocarpine concentration was applied (Fig. 1A-D). Since hepatocyte cell death is the primary cause of septic liver dysfunction (2), liver histology was subsequently analyzed by $H \& E$ staining. The structure of the hepatic lobules was found to be preserved in groups treated with sophocarpine and damaged in those from the CLP groups (Fig. 1E). TUNEL assay, which was applied to measure the extent of cell death, also demonstrated that sophocarpine attenuated hepatocyte death (Fig. 1F), as increased brown staining indicates increased cell death. These results suggested that treatment with sophocarpine may attenuate septic liver injury.

Sophocarpine attenuates NLRP3 inflammasome activation following CLP-induced liver injury. Previous studies have suggested that inflammasome activation, especially that of the NLRP3 inflammasome, serves a key role in liver injury $(9,11,19)$, resulting in the release of IL-1 $\beta$ from hepatocytes and subsequent pyroptosis $(6,11)$. Therefore, following the CLP procedure and/or sophocarpine treatment, the mice were sacrificed and the liver was harvested, following which the extent of NLRP3 activation was assessed. NLRP3 inflammasome was found to be highly activated in the CLP group, as indicated by the elevated NLRP3 expression, activated caspase 1-p20 and elevated GSDMD-p30 (GSDMD-p30) levels compared with those in the NC group (Fig. $2 \mathrm{~A}$ ). IL-1 $\beta$ levels in the liver was also found to be elevated in the CLP groups compared with those in the NC group (Fig. 2B). However, compared with that in the CLP group, activation of caspase 1 and GSDMD was found to be attenuated in the sophocarpine-treated group, with reduced levels of liver IL-1 $\beta$ also observed (Fig. 2A and B). To assess NLRP3 inflammasome activation further, IFHC was used to study NLRP3, an indicator for inflammasome activation (4) in the liver. The results indicated that although CLP promoted NLRP3 

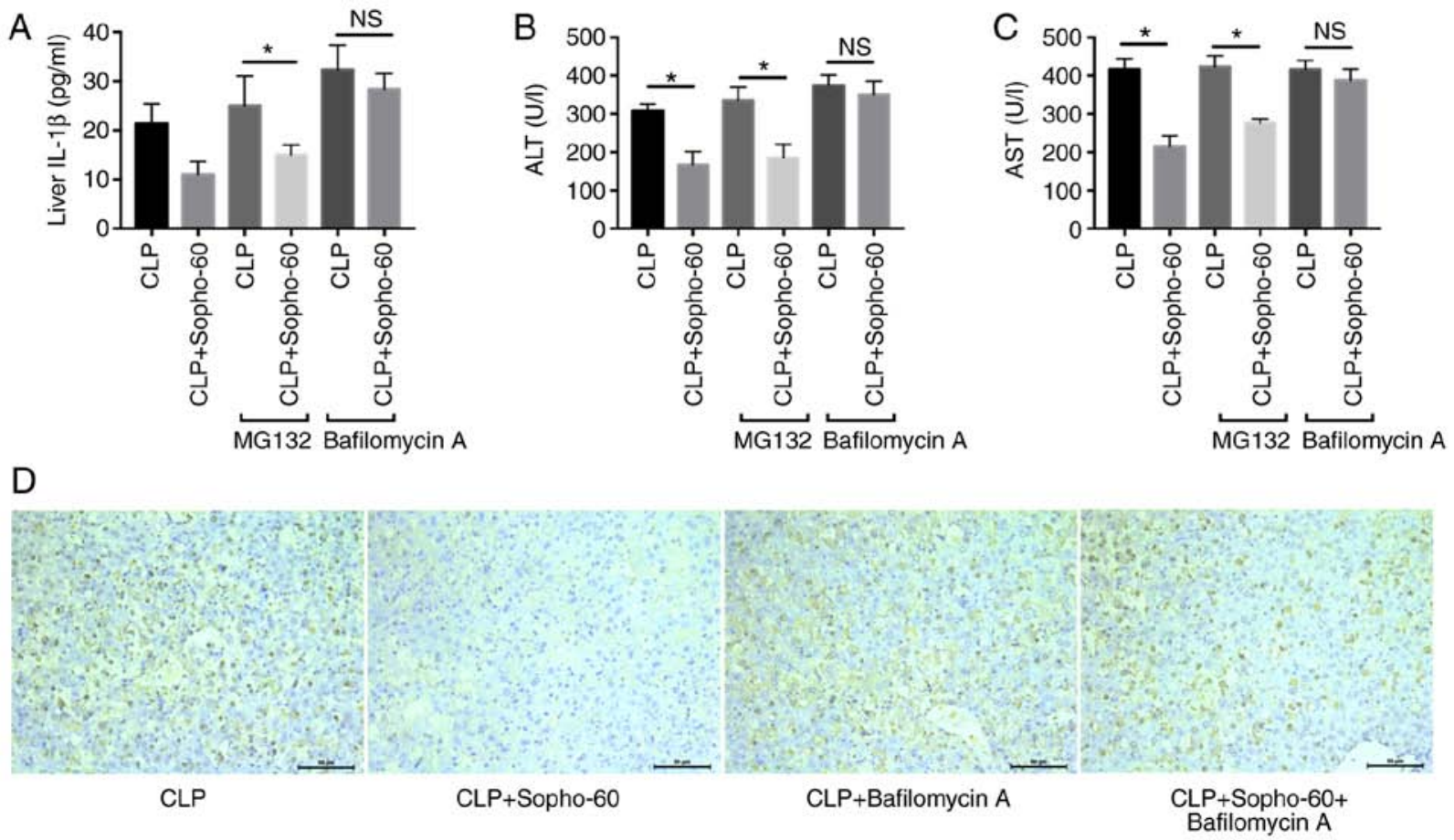

Figure 3. Autophagy is essential for sophocarpine-mediated attenuation of CLP-induced liver injury. (A) Liver IL-1 $\beta$, (B) serum ALT and (C) AST levels in mice treated with MG132, Bafilomycin A or CLP group. $n=5$ mice per group. (D) TUNEL staining of liver tissues isolated from mice that underwent CLP followed by Sopho with or without Bafilomycin A treatment. Scale bar, $50 \mu \mathrm{m}{ }^{*} \mathrm{P}<0.05$. ALT, alanine transaminase; AST, aspartame transaminase; CLP, cecal ligation and puncture; IL, interleukin; Sopho- 60 , sophocarpine treatment $60 \mathrm{mg} / \mathrm{kg}$.

activation in the liver in comparison to sham, this was reversed by sophocarpine treatment (Fig. 2C). Together, these results suggested that sophocarpine attenuated NLRP3 inflammasome activation following CLP-induced liver injury.

Autophagy is essential for sophocarpine-mediated attenuation of NLRP3 inflammasome activation following CLP-induced liver injury. A previous study has indicated that sophocarpine may promote autophagy in gastric cancer cells (20). Additionally, the NLRP3 inflammasome may be degraded by the proteasome or the autophagic process in macrophage (21). Therefore, the route by which the inflammasome is degraded during sophocarpine-induced attenuation of liver injury was assessed. As the results indicated, pre-treatment with MG-132 did not influence the reduction in AST and ALT as a result of sophocarpine treatment, whilst bafilomycin A significantly reversed the protective effects of sophocarpine in CLP-induced liver injury (Fig. 3B and C). In addition, the levels of liver IL-1 $\beta$ also showed a similar tendency, in that autophagy inhibition by bafilomycin A increased liver IL-1 $\beta$ levels in the sophocarpine-treated group compared with those in the CLP group (Fig. 3A). These results suggested that autophagy is essential for the protective effect of sophocarpine in CLP-induced liver injury.

The effect of autophagy on sophocarpine-mediated NLRP3 inflammasome activation was subsequently investigated by western blotting. Following the induction of CLP-induced liver injury, sophocarpine attenuated the activation of NLRP3, caspase 1-p20 and GSDMD-p30 compared with that in the CLP group (Fig. 4A). By contrast following liver injury induction, In mice treated with bafilomycin A, liver NLRP3 expression and caspase 1-p20 and GSDMD-p30 activation were found to be expressed at markedly higher levels in the presence of sophocarpine compared with mice treated with sophocarpine alone (Fig. 4A). These results indicated that inhibition of autophagy restored NLRP3 inflammasome activation following liver injury induction, suggesting that autophagy is essential for the sophocarpine-mediated attenuation of NLRP3 inflammasome activation during CLP-induced liver injury. Sophocarpine was further suggested to promote the autophagy process, through the observed elevation in LC3 levels and reduction in p62 expression in mouse livers of mice following CLP-induced liver injury compared with untreated mice following CLP-induced liver injury (Fig. 4B). These results suggested that sophocarpine may attenuate NLRP3 inflammasome activation by promoting its degradation by autophagy, thereby exhibiting protective effects against CLP-induced liver injury.

\section{Discussion}

The updated definition of sepsis highlights organ dysfunction as the primary cause of mortality associated with sepsis $(1,3)$. Uncontrolled inflammation and pathogen invasion leads to exacerbated immune responses and activation of the cytokine storm (1). The cytokines released, including IL-1 $\beta$ and IL-6, bind to receptors on hepatocytes and initiate cellular stress responses (2-4). Although recent research suggest a potential protective effect of sophocarpine on autoimmune hepatitis (14), LPS-induced liver injury (13), lupus nephritis (10) and arthritis (15), the possible effects of sophocarpine on septic liver injury remain poorly elucidated. Results from the present 


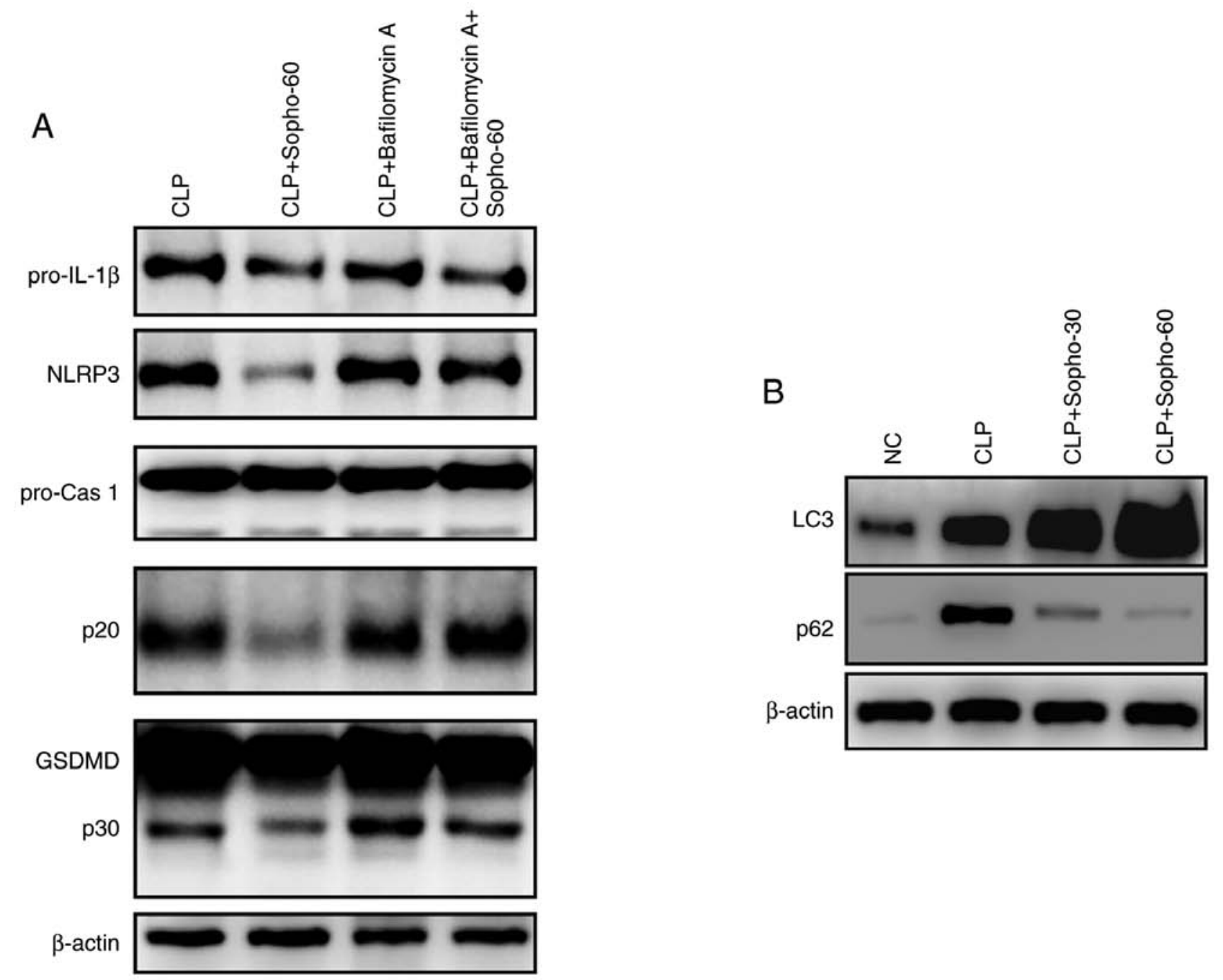

Figure 4. Autophagy is essential for the sophocarpine-mediated attenuation of NLRP3 inflammasome activation in CLP-induced liver injury. (A) Representative western blotting images of pro-IL-1 $\beta$, NLRP3, pro-caspase 1 and p20, GSDMD and p30 and $\beta$-actin. (B) Representative western blotting images of LC3 and p62 in liver tissues isolated from mice that underwent CLP followed by Sopho with or without Bafilomycin A treatment. CLP, cecal ligation and puncture; cas, caspase; GSDMD, gasdermin D; IL, interleukin; NC, normal control; Sopho-30, sophocarpine treatment $30 \mathrm{mg} / \mathrm{kg}$; Sopho-60, sophocarpine treatment $60 \mathrm{mg} / \mathrm{kg}$; NLRP3, nucleotide-binding oligomerization domain, leucine rich repeat and pyrin domain containing 3.

study suggested a protective effect of sophocarpine against CLP-induced liver injury, as sophocarpine treatment resulted in reduced AST and ALT levels in the serum and suppression of hepatocyte cell death compared with those in untreated mice following CLP. Previous studies have applied sophocarpine as a pre-treatment for liver injury or LPS-induced inflammation, typically 5-7 days prior to model establishment (10-11,13-14). In the present study, sophocarpine was applied 2 days before the CLP procedure and continuously applied thereafter for 3 days to investigate their possible therapeutic effects as a perioperative medication for clinical application.

Previous studies have demonstrated that hepatocyte death through mechanisms including apoptosis, necrosis and autophagy, serves a crucial role in septic liver injury (3-5). The death of vast numbers of hepatocytes leads to elevated levels of AST and ALT and subsequent liver failure (7). In addition, pyroptosis, a newly discovered mechanism of cell death initiated by inflammasome activation, has been revealed to be an essential mechanism for liver injury and dysfunction in a number of liver injury models $(6,11)$. It has been reported that the activation of inflammasome in hepatocytes participates in acute hepatic conditions such as drug-induced liver injury and acute hepatitis (22), in addition to chronic liver conditions such as liver fibrosis and cancer $(23,24)$. The majority of studies have identified the NLRP3 inflammasome as the main type of inflammasome implicated in liver injury $(7,9,11,25)$. During cellular stress or inflammation, activated NLRP3, together with other inflammasome elements such as apoptosis-associated speck-like protein containing a CARD and pro-caspase 1, assemble to form the mature NLRP3 inflammasome (11). Pro-caspase 1 becomes activated as a result, to become cleaved caspase 1-p20 which then induces the cleavage of IL-1 and GSDMD (26). Cleaved GSDMD-p30 subsequently forms a membrane pore and releases IL-1 $\beta$, leading to the subsequent pyroptosis of hepatocytes (26). In the present study, sophocarpine suppressed the activation of the NLRP3 inflammasome, as indicated by the reduced levels of caspase 1-p20, GSDMD-p30, IL-1 $\beta$ and the expression of NLRP3 in liver tissues. These results indicated sophocarpine may alleviate the pyroptosis of hepatocytes during septic liver injury, suggesting 
that inflammasome attenuation may be an important target of sophocarpine.

Regulation of the NLRP3 inflammasome activation status is mediated by the regulation of its degradation. In addition to the regulation of complex assembly, based on the 'two signal' theory, during the activation phase of the inflammasome $(21,23,27)$, degradation of NLRP3 also serves an essential role in determining the fate of inflammasome activation (21). It has been reported that NLRP3 is typically degraded through the autophagy or the proteasome pathway $(17,21)$. Since sophocarpine was previously reported to promote autophagy (20), it was hypothesized in the present study that sophocarpine may regulate NLRP3 inflammasome activation by degradation via the autophagy pathway. The present study suggested that the inhibitor of autophagy, bafilomycin A, significantly reversed the protective effects of sophocarpine in CLP-induced liver injury, whilst the proteasome inhibitor, MG-132, exerted little effects. Following treatment with bafilomycin A, the inhibition of NLRP3 inflammasome activation by sophocarpine was also found to be reversed, suggesting that autophagy serve as the major mechanism in inflammasome inhibition by sophocarpine.

There are some limitations associated with the present study. Firstly, the protocol for the application of sophocarpine require a more comprehensive investigation. The present study adopted a short-term pre-treatment strategy combined with post-treatment, which uncovered the protective effects of sophocarpine. Future studies may adopt a single post-treatment approach in a specific disease model to evaluate its effects, which may provide superior indications for future clinical applications. Although sophocarpine was found to promote NLRP3 inflammasome degradation through autophagy, the possible effects of sophocarpine during the activation phase should be addressed. Studies into the regulation of inflammasome activation have focused on the two-signal mechanism and have unveiled several important regulatory pathways, where reactive oxygen species have been discovered to act as an important signaling molecule (21). A previous study also suggested that sophocarpine could alleviate oxidative stress in hepatocytes (13). Therefore, it is reasonable to speculate that sophocarpine may also regulate the activation of NLRP3 inflammasome.

Collectively, the results of the present study indicated that sophocarpine could attenuate septic liver injury and NLRP3 inflammasome activation in hepatocytes. Sophocarpine-induced NLRP3 degradation by autophagy may be beneficial in the alleviation of septic liver injury.

\section{Acknowledgements}

Not applicable.

\section{Funding}

The current study was supported by the Research Program of Qingdao Municipal Hospital (grant no. QD20190223).

\section{Availability of data and materials}

The datasets used and/or analyzed during the current study are available from the corresponding author on reasonable request.

\section{Authors' contributions}

NH performed the majority of the experiments and prepared the manuscript. XD, WL and HYa assisted with the CLP procedures and contributed to the western blotting analysis. $\mathrm{HYu}, \mathrm{JL}, \mathrm{HL}$ and XS assisted with animal preparation and contributed to manuscript preparation. DA designed the study and reviewed the manuscript. All authors read and approved the final manuscript.

\section{Ethics approval and consent to participate}

Animal experiments in the present study were approved by the Ethics Committee of Qingdao Municipal Hospital.

\section{Patient consent for publication}

Not applicable.

\section{Competing interests}

The authors declare that they have no competing interests.

\section{References}

1. Singer M, Deutschman CS, Seymour CW, Hari MS, Annane D, Bauer M, Bellomo R, Bernard GR, Chiche JD, Coopersmith CM, et al: The Third International Consensus Definitions for Sepsis and Septic Shock (Sepsis-3). Jama 315: 801-810, 2016.

2. Strnad P, Tacke F, Koch A and Trautwein C: Liver-guardian, modifier and target of sepsis. Nat Rev Gastroenterol Hepatol 14: 55-66, 2016.

3. Yan J, Li S and Li S: The role of the liver in sepsis. Int Rev Immunol 33: 498-510, 2014

4. Savio LEB, de Andrade Mello P, Figliuolo VR, de Avelar Almeida TF, Santana PT, Oliveira SDS, Silva CLM, Feldbrügge L, Csizmadia E, Minshall RD, et al: CD39 limits $\mathrm{P} 2 \mathrm{X} 7$ receptor inflammatory signaling and attenuates sepsis-induced liver injury. J Hepatol 67: 716-726, 2017.

5. Alhusaini A, Faddaa L, Ali HM, Hassan I, El Orabi NF and Bassiouni Y: Amelioration of the protein expression of Cox 2 $\mathrm{NF} \kappa \mathrm{B}$, and STAT-3 by some antioxidants in the liver of sodium fluoride-intoxicated rats. Dose-Response 16: 1559325818800153 , 2018.

6. Geng Y, Ma Q, Liu YN, Peng N, Yuan FF, Li XG, Li M, Wu YS, Li BL, Song WB, et al: Heatstroke induces liver injury via IL-1 $\beta$ and HMGB1-induced pyroptosis. J Hepatol 63: 622-633, 2015.

7. Lebeaupin C, Proics E, de Bieville CH, Rousseau D, Bonnafous S, Patouraux S, Adam G, Lavallard VJ, Rovere C, Le Thuc O, et al: ER stress induces NLRP3 inflammasome activation and hepatocyte death. Cell Death Dis 6: e1879-e1879, 2015.

8. Li W, Li Y, Siraj S, Jin H, Fan Y, Yang X, Huang X, Wang X, Wang J, Liu L, et al: FUN14 domain-containing 1-mediated mitophagy suppresses hepatocarcinogenesis by inhibition of inflammasome activation in mice. Hepatology 69: 604-621, 2019.

9. Han CY, Rho HS, Kim A, Kim TH, Jang K, Jun DW, Kim JW, Kim B and Kim SG: FXR inhibits endoplasmic reticulum stress-induced NLRP3 inflammasome in hepatocytes and ameliorates liver injury. Cell Rep 24: 2985-2999, 2018.

10. Li X, Wang M, Hong H, Luo C, Liu Z and Yang R: Sophocarpine attenuates murine lupus nephritis via inhibiting NLRP3 inflammasome and NF- $\kappa$ B activation. Immunol Res 66: 521-527, 2018.

11. Zhang X, Luan J, Chen W, Fan J, Nan Y, Wang Y, Liang Y, Meng G and Ju D: Mesoporous silica nanoparticles induced hepatotoxicity via NLRP3 inflammasome activation and caspase-1-dependent pyroptosis. Nanoscale 10: 9141-9152, 2018.

12. Yang CS, Kim JJ, Kim TS, Lee PY, Kim SY, Lee HM, Shin DM, Nguyen LT, Lee MS, Jin HS, et al: Small heterodimer partner interacts with NLRP3 and negatively regulates activation of the NLRP3 inflammasome. Nature Commun 6: 6115, 2015. 
13. Jiang Z, Meng Y, Bo L, Wang C, Bian J and Deng X: Sophocarpine attenuates LPS-induced liver injury and improves survival of mice through suppressing oxidative stress, inflammation, and apoptosis. Mediators Inflamm 2018: 5871431, 2018.

14. Sang XX, Wang RL, Zhang CE, Liu SJ, Shen HH, Guo YM, Zhang YM, Niu M, Wang JB, Bai ZF and Xiao XH: Sophocarpine protects mice from ConA-induced hepatitis via inhibition of the IFN-Gamma/STAT1 pathway. Front Pharmacol 8: 140, 2017.

15. Zhu L and Zhu L: Sophocarpine suppress inflammatory response in human fibroblast-like synoviocytes and in mice with collagen-induced arthritis. Eur Cytokine Netw 28: 120-126, 2017.

16. Jiang Z, Bo L, Meng Y, Wang C, Chen T, Wang C, Yu X and Deng $\mathrm{X}$ : Overexpression of homeodomain-interacting protein kinase 2 (HIPK2) attenuates sepsis-mediated liver injury by restoring autophagy. Cell Death Dis 9: 847, 2018.

17. Harhouri K, Navarro C, Depetris D, Mattei MG, Nissan X, Cau P, De Sandre-Giovannoli A and Lévy N: MG132-induced progerin clearance is mediated by autophagy activation and splicing regulation. EMBO Mol Med 9: 1294-1313, 2017.

18. Roustan A, Perrin J, Berthelot-Ricou A, Lopez E, Botta A and Courbiere B: Evaluating methods of mouse euthanasia on the oocyte quality: Cervical dislocation versus isoflurane inhalation Lab Anim 46: 167-169, 2012.

19. Gehrke N, Hövelmeyer N, Waisman A, Straub BK, Weinmann-Menke J, Wörns MA, Galle PR and Schattenberg JM: Hepatocyte-specific deletion of IL1-RI attenuates liver injury by blocking IL-1 driven autoinflammation. J Hepatol 68: 986-995, 2018.

20. Huang Y, Chen X, Guo G, Guo W, Ma Q and Yuan J: Sophocarpine inhibits the growth of gastric cancer cells via autophagy and apoptosis. Front Biosci (Landmark Ed) 24: 616-627, 2019.
21. Xu M, Jiang Z, Wang C, Li N, Bo L, Zha Y, Bian J, Zhang Y and Deng X: Acetate attenuates inflammasome activation through GPR43-mediated $\mathrm{Ca}^{2+}$-dependent NLRP3 ubiquitination. Exp Mol Med 51: 1, 2019.

22. Woolbright BL and Jaeschke H: Role of the inflammasome in acetaminophen-induced liver injury and acute liver failure. J Hepatol 66: 836-848, 2017.

23. Luan J and Ju D: Inflammasome: A double-edged sword in liver diseases. Front Immunol 9: 2201, 2018.

24. Alegre F, Pelegrin P and Feldstein AE: Inflammasomes in liver fibrosis. Semin Liver Dis 37: 119-127, 2017.

25. Zheng T, Yang X, Li W, Chen L, Wu Dan, Bian F, Xing S and Jin S: Salidroside attenuates high-fat diet-induced nonalcoholic fatty liver disease via AMPK-dependent TXNIP/NLRP3 pathway. Oxid Med Cell Longev 2018: 8597897, 2018

26. Shi J, Zhao Y, Wang K, Shi X, Wang Y, Huang H, Zhuang Y, Cai Tao, Wang F and Shao F: Cleavage of GSDMD by inflammatory caspases determines pyroptotic cell death. Nature 526: 660-665, 2015

27. Kayagaki N, Stowe IB, Lee BL, O'Rourke K, Anderson K, Warming S, Cuellar T, Haley B, Roose-Girma M, Phung QT, et al: Caspase-11 cleaves gasdermin D for non-canonical inflammasome signalling. Nature 526: 666-671, 2015.

This work is licensed under a Creative Commons Attribution-NonCommercial-NoDerivatives 4.0 International (CC BY-NC-ND 4.0) License. 